\title{
Eficacia de biocontroladores y fungicidas para el control de Pythium sp. en crisantemo (Dendranthema grandiflora (Ramat.) Kitam.)
}

\section{Efficacy of biocontrollers and fungicides for the control of Pythium sp. in chrysanthemum (Dendranthema grandiflora (Ramat.) Kitam.)}
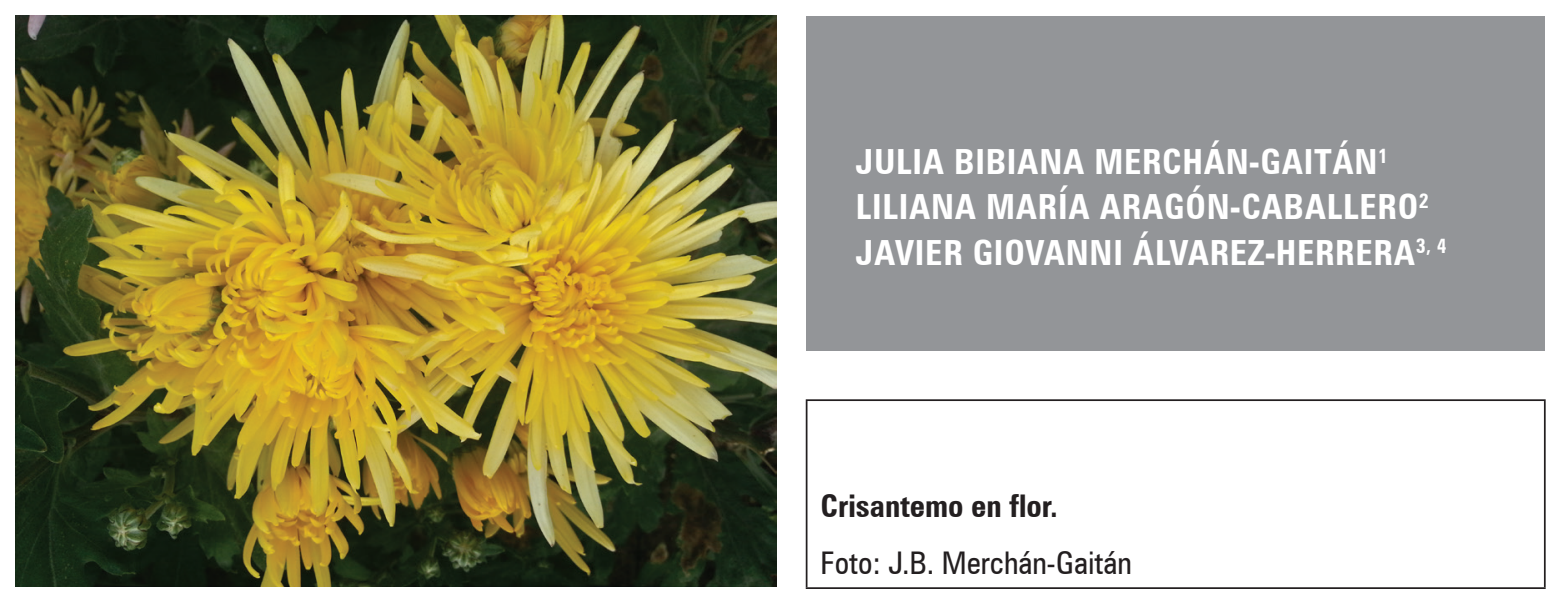

\section{RESUMEN}

Dentro de las enfermedades que se presentan en la producción de crisantemo a nivel mundial, una de las más importantes es la pudrición de raíz causada por Pythium sp. por lo anterior, se realizó una investigación cuyo objetivo fue evaluar la dosis óptima de biocontroladores y fungicidas químicos sobre Pythium sp. La aplicación de los tratamientos se llevó a cabo tanto en laboratorio como en el cultivo bajo invernadero. Se empleó un diseño completamente al azar con 10 tratamientos (testigo, metalaxil y clorotalonil, azoxystrobin, Trichoderma harzianum, Bacillus subtilis, aplicados en dos dosis 100\% de la aplicación comercial y 150\%). Los resultados obtenidos en la fase de laboratorio mostraron que el testigo inoculado con oosporas tuvo el mayor crecimiento micelial de Pythium sp., $36,43 \mathrm{~cm}$ en diámetro, así mismo los tratamientos con fungicidas químicos presentaron un control del $100 \%$ ya que no hubo crecimiento de Pythium sp. En el invernadero, las plantas de crisantemo mostraron los mejores resultados cuando se aplicó T. harzianum en dosis de $150 \%$, ya que se presentó tan solo un $9,33 \%$ de incidencia, mientras que referente a la severidad, el tratamiento más eficaz fue con T. harzianum en dosis comercial con 4,9\%. El mejor comportamiento de las características agronómicas de las plantas de crisantemo se presentó con la aplicación de T. harzianum con dosis de 150\%, ya que las plantas mostraron mayor altura, masa radicular fresca y seca, masa aérea fresca y seca, número de botones, diámetro floral y masa floral.

\footnotetext{
Universidad de Tarapacá, Facultad de Educación, Iquique (Chile). ORCID Merchán-Gaitán, J.B.: 0000-0002-2753-0306

2 Universidad Nacional Agraria La Molina (UNALM), Facultad de Agronomía, Departamento de Fitopatología, Lima (Perú). ORCID Aragón-Caballero, L.M.: 0000-0003-0312-5020

3 Universidad Pedagógica y Tecnológica de Colombia (UPTC), Facultad de Ciencias Agropecuarias, Grupo de Investigaciones Agrícolas, Tunja (Colombia). ORCID Álvarez-Herrera, J.G.: 0000-0002-1737-6325

4 Autor de correspondencia. javier.alvarez@uptc.edu.co
} 


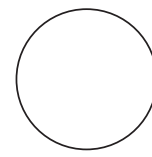

Palabras clave adicionales: Trichoderma harzianum; Bacillus subtilis; pudrición radicular; fungicidas; calidad de flor; contrastes ortogonales.

\begin{abstract}
Damping off is caused by Pythium sp. in seedlings, which is the main cause of losses in chrysanthemum crops. This research aimed to evaluate the optimal dose of biocontrollers and chemical fungicides for Pythium sp. The application of the treatments was carried out both in a laboratory and on a greenhouse crop. A completely randomized design was used with 10 treatments (control, metalaxyl and chlorothalonil, azoxystrobin, Trichoderma harzianum, and Bacillus subtilis), applied at two doses: $100 \%$ and $150 \%$ of the commercial application. The results obtained in the laboratory phase showed that the inoculated control had the highest mycelial growth of Pythium sp., $36.43 \mathrm{~cm}$ in diameter. The treatments with chemical fungicides presented $100 \%$ control since there was no growth of Pythium $\mathrm{sp}$. In the greenhouse, the chrysanthemum cultivation had the best results when $T$. harzianum was applied at a dose of $150 \%$ since only $9.33 \%$ incidence was observed. In terms of severity, the most effective treatment was the commercial dose of $T$. harzianum, with $4.9 \%$. The best performance of the agronomic characteristics in the chrysanthemum plants was seen with the application of T. harzianum at a dose of $150 \%$ because the plants presented higher plant height, fresh radicular mass, dry root mass, fresh aerial mass, mass dry air, number of buttons, floral diameter and floral mass.
\end{abstract}

Additional key words: Trichoderma harzianum; Bacillus subtilis; root rot; fungicides; flower quality; orthogonal contrasts.

Fecha de recepción: 24-04-2018 Aprobado para publicación: 30-11-2018

INTRODUCCIÓN

Uno de los cultivos que actualmente genera mayores divisas dentro de las exportaciones no tradicionales colombianas es la floricultura. En un período menor de 10 años, Colombia se ha convertido en el segundo exportador mundial (después de Holanda) de flores cortadas, con una participación del $10 \%$ sobre el total de las exportaciones mundiales. Del mismo modo, en Perú es un cultivo en auge y existen aproximadamente 2.000 productores dedicados a la siembra de flores en 971 ha. El uso de crisantemos como plantas decorativas en jardines e interiores ha tomado gran importancia en estos países, sin embargo, uno de los principales problemas en la producción de estas flores es el daño fitosanitario que ocasionan las enfermedades causadas por Pythium sp. las cuales afectan todas las etapas del ciclo de producción, ya que el patógeno es capaz de sobrevivir en agua, residuos de plantas y suelo.

Sin embargo, algunas especies de Pythium como $P$. irregulare, $P$. intermedium y $P$. graminicola tienen la capacidad de atacar plantas de crisantemo y pueden sobrevivir saprofíticamente sobre residuos de tejido muerto vegetal y otras especies pueden ser patogénicas, causando pudriciones y finalmente la muerte de las plantas en diversos cultivos (León, 2017). De esta manera, es posible la introducción o el incremento de algunos microorganismos antagonistas, los cuales tienen la capacidad de inactivar los propágalos (oosporas y zoosporas) del patógeno, reducir su número o afectar el desarrollo de la enfermedad (Rinaudo et al., 2010). Por lo anterior, el objetivo del presente trabajo fue evaluar la efectividad de biocontroladores y fungicidas en el control de Pythium sp. en crisantemo (Dendranthema grandiflora (Ramat.) Kitam., syn. Chrysanthemum morifolium Ramat.).

\section{MATERIALES Y MÉTODOS}

El estudio in vitro y los análisis se realizaron en el laboratorio de fitopatología de la Universidad Nacional Agraria La Molina (Lima, Perú).

Identificación del Pythium sp.: se colocaron 10 discos de $5 \mathrm{~mm}$ de radio que contenían micelio del patógeno 
en 10 cajas Petri (un disco por caja) con una solución patrón de suelo diluido en agua destilada estéril en 10 $\mathrm{g}$ de suelo y se disolvió bajo condiciones de asepsia en $100 \mathrm{~mL}$ de agua destilada estéril, y a partir de la solución anterior se prepararon soluciones más diluidas de concentraciones hasta $10^{-6}$ (solución suelo esterilizado), para inducir la formación de zoosporangios. Después de que se formaron los esporangios, se indujo la formación de zoosporas mediante tratamiento en frío, para ello las placas fueron ubicadas dentro de una nevera a una temperatura de $4^{\circ} \mathrm{C}$ durante una hora siguiendo la metodología descrita por Dhingra y Sinclair (1995). La identificación probable de la especie corresponde a Pythium aphanidermatum la cual se efectuó mediante la observación al microscopio óptico (40X) con tinción de azul de lactofenol; se determinó la morfología del micelio, tipo de esporangio, oospora, anteridio y zoospora, (Fig. 1) mediante el uso de la clave de Van der Plaats-Niterink (1981).

Se realizó el método de medio envenenado sugerido por Dhingra y Sinclair (1995) con los productos biológicos y químicos, se pesó cada producto de acuerdo a las dosis establecidas en los tratamientos (Tab. 1) para lo cual se añadió al medio harina de maíz Agar (CMA; CRITERIO ${ }^{\mathrm{TM}}$ ), una vez homogenizados, se realizó el servido del medio correspondiente, colocándose con ayuda de un sacabocado un disco de $5 \mathrm{~mm}$

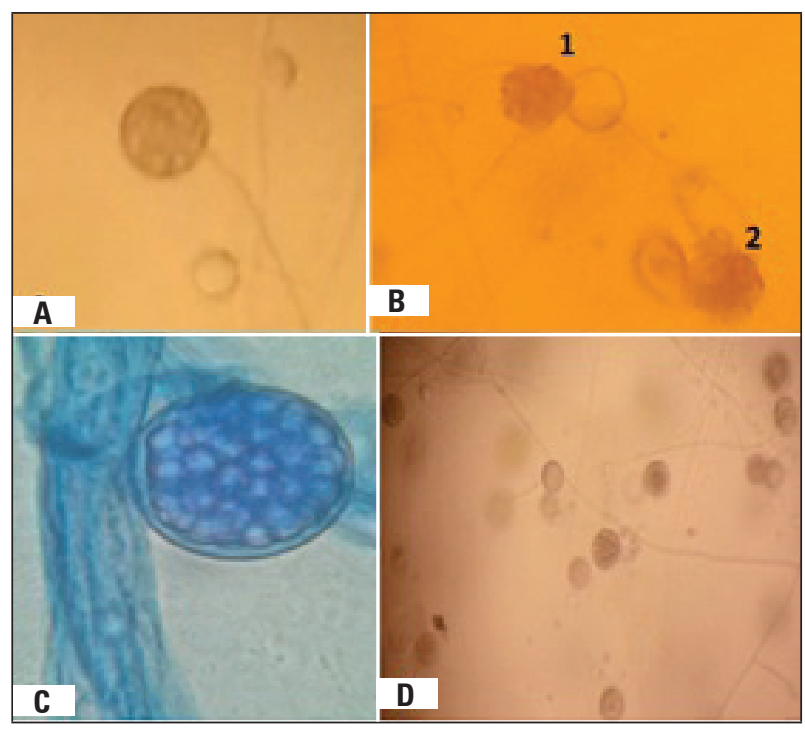

Figura 1. Estructuras de Pythium posiblemente de la especie aphanidermatum. A. Oogonio. B1. Zoosporangio globoso y vacío con la vesícula que contiene zoosporas, B2. Liberación de zoosporas de la vesícula. C. Zoosporangio conteniendo la vesícula con diferentes zoosporas a $100 \mathrm{X}$, D. Micelio y oosporas. de diámetro procedente de cada uno de los cultivos del fitopatógeno en el centro de la superficie de la caja Petri, la cual contenía $10 \mathrm{~mL}$ del medio. Las cajas sembradas tenían un tamaño de $10 \mathrm{~cm}$ de diámetro y fueron incubadas a $25^{\circ} \mathrm{C}$ por $4 \mathrm{~d}$. El crecimiento micelial del Pythium sp. se midió a intervalos de $24 \mathrm{~h}$, ajustándose a 90 cajas Petri utilizadas en las pruebas del medio envenenado.

El cultivo fue sembrado bajo condiciones controladas en el invernadero de estructura metálica y vidrio de la Universidad Nacional Agraria La Molina, a una temperatura promedio de $22^{\circ} \mathrm{C}$ y una humedad de $75 \%$. El material vegetal usado fue esquejes de crisantemo (Dendranthema grandiflora (Ramat.) Kitam., syn. Chrysanthemum morifolium Ramat.). de 20 d de enraizados, variedad Anastasia.

Para inocular las plántulas de crisantemo en invernadero, se limpiaron y se embolsaron $10 \mathrm{~g}$ de trigo, luego se esterilizó en autoclaves a una temperatura de $121^{\circ} \mathrm{C}$ durante $30 \mathrm{~min}$. Concluida la esterilización se dejó enfriar a temperatura ambiente en una cámara de flujo laminar, luego se extrajo desde una caja Petri varios trozos de $5 \mathrm{~cm}$ por $5 \mathrm{~cm}$ de CMA con micelio del seudohongo y se inoculó en cada bolsa, dejándose entre 10 a 15 d para que tuviera una colonización completa del fitopatógeno por el método sugerido por Drenth y Sendall (2001). Luego se inoculó $10 \mathrm{~g}$ de trigo, colonizado con Pythium sp., en el suelo de las macetas en hoyos a una profundidad de $10 \mathrm{~cm}$ para estar más cerca de la raíz y $5 \mathrm{~cm}$ lejos del tallo a los 40 días después de sembrado los esquejes de plantas de crisantemo.

El diseño experimental fue completamente al azar, con 10 tratamientos (Tab. 1) y cinco repeticiones. En la fase in vitro se omitió el tratamiento 1 (testigo). Cada repetición se hizo en dos placas, para un total de 90 placas. En la fase de cultivo en invernadero, se tuvieron 50 unidades experimentales (UE) y cada UE contó con tres plantas, para un total de 150.

Las variables de respuesta evaluadas en laboratorio fueron, incidencia de la enfermedad, la cual se evaluó todos los días, se observó si la planta mostraba síntomas de pudrición y ahogamiento por lo cual fue considerada como planta enferma. La evaluación se realizó de acuerdo con el número de plantas que mostraron los síntomas de la enfermedad en relación al número de plantas totales, expresadas en porcentaje; para la severidad se calculó el área afectada de cada raíz de acuerdo a la escala propuesta por Henfling 


\begin{tabular}{|c|c|c|c|}
\hline Tratamiento & Ingrediente activo (Nombre comercial) & $\begin{array}{c}\text { Dosis } \\
(2,5 \mathrm{~kg} \text { de suelo }) / \text { planta }\end{array}$ & $\begin{array}{c}\text { Dosis } \\
\text { comercial (\%) }\end{array}$ \\
\hline $\mathrm{T} 1^{*}$ & Testigo & Sin inóculo & 0 \\
\hline T2 & Testigo & Inoculado con Pythium (IP) & (10 g de inóculo /maceta) \\
\hline T3 & Metalaxil y Clorotalonil (Folio Gold®440 SC) & $0,015 \mathrm{~mL}$ en $0,35 \mathrm{Ml}$ de agua $+(\mathbb{P})$ & 100 \\
\hline T4 & Metalaxil y Clorotalonil (Folio Gold®440 SC) & $0,022 \mathrm{~mL}$ en $0,525 \mathrm{~mL}$ de agua $+(\mathrm{IP})$ & 150 \\
\hline T5 & Azoxystrobin (Amistar ${ }^{\circledR} 50$ WG) & $0,023 \mathrm{~g}$ en $58,66 \mathrm{~mL}$ de agua $+(\mathrm{IP})$ & 100 \\
\hline T6 & Azoxystrobin (Amistar ${ }^{\circledR} 50$ WG) & 0,0352 g en $117 \mathrm{~mL}$ de agua + (IP) & 150 \\
\hline $\mathrm{T7}$ & Trichoderma harzianum (Tricho D® WP) & 0,007 g en $134 \mathrm{~mL}$ de agua $+(\mathrm{IP})$ & $100\left(1 \times 10^{8}\right.$ esporas/g) \\
\hline T8 & Trichoderma harzianum (Tricho D® WP) & 0,01 g en $201 \mathrm{~mL}$ de agua $+($ IP) & 150 \\
\hline T9 & Bacillus subtilis Cepa AP-01 (Bio-Splent ${ }^{\circledR} 70$ WP) & $0,0088 \mathrm{~g}$ en $1,17 \mathrm{~mL}$ de agua $+(\mathrm{IP})$ & $100\left(1 \times 10^{9} \mathrm{UFC} / \mathrm{g}\right)$ \\
\hline $\mathrm{T} 10$ & Bacillus subtilis Cepa AP-01 (Bio-Splent® 70 WP) & $0,0132 \mathrm{~g}$ en $1,755 \mathrm{~mL}$ de agua $+(\mathrm{IP})$ & 150 \\
\hline
\end{tabular}

*Este tratamiento no se realizó en la fase de laboratorio. UFC: unidades formadoras de colonia

(1982), modificada y adaptada para crisantemo (Tab. 2). El grado 0 equivale a una coloración clara de raíz, indicando que no hay presencia de pudrición, es decir una planta totalmente sana y el grado 6 a una planta con la raíz de coloración oscura, indicando presencia de pudrición en un $100 \%$.

\begin{tabular}{|c|c|c|}
\hline Tabla 2. & $\begin{array}{c}\text { Escala para la evaluación de severidad en el siste- } \\
\text { ma radicular en plantas de crisantemo. }\end{array}$ \\
\hline Grado & $\begin{array}{c}\text { Síntomas en el sistema } \\
\text { radicular (\%) }\end{array}$ & Coloración \\
\hline 0 & 0 & \\
\hline 1 & $1-20$ & \\
\hline 2 & $21-40$ & \\
\hline 3 & $41-60$ & \\
\hline 4 & $61-80$ & \\
\hline 5 & $81-99$ & \\
\hline 6 & 100 & \\
\hline
\end{tabular}

La altura de las plantas de crisantemo se midió mediante el uso de una cinta métrica desde el cuello hasta el ápice terminal, después de la aplicación de los tratamientos una vez a la semana, durante 4 meses. La masa fresca y seca radicular y de la parte aérea se determinó mediante una balanza digital Henkel con precisión $0,001 \mathrm{~g}$. También se contó el número de botones florales para estimar la producción de cada tallo por UE. Se midió el diámetro de la flor con un calibrador vernier digital Mitutoyo Digimattic CD 6" CSX y se determinó la masa floral por planta al momento de la cosecha.

A los datos se les realizó un análisis de varianza (Anova), prueba de Tukey y luego una prueba de contrastes ortogonales (Tab. 3) con el fin de determinar las diferencias entre tratamientos a un nivel de confiabilidad del 95\% ( $P \leq 0,05)$, mediante el paquete estadístico SAS v.8e. (SAS Institute Inc., Cary, NC).

Tabla 3. Evaluación de los contrastes ortogonales realizados en la fase de laboratorio y de invernadero.

\begin{tabular}{|c|l|}
\hline Agrupación & \multicolumn{1}{|c|}{ Comparaciones } \\
\hline $1^{*}$ & Con aplicación de control vs. Sin aplicación \\
\hline 2 & Testigo sin Pythium sp. vs. Testigo con Pythium sp. \\
\hline $3^{*}$ & Tratamientos químicos vs. Tratamientos biológicos \\
\hline 4 & Metalaxil + clorotalonil vs. Azoxystrobin \\
\hline 5 & $\begin{array}{l}\text { Azoxystrobin dosis } 100 \% \text { vs. Azoxystrobin dosis } \\
150 \%\end{array}$ \\
\hline 6 & $\begin{array}{l}\text { Metalaxil + clorotalonil dosis } 100 \% \text { vs. Metalaxil }+ \\
\text { clorotalonil dosis } 150 \%\end{array}$ \\
\hline $7^{*}$ & Trichoderma harzianum vs. Bacillus subtilis \\
\hline 8 & T. harzianum dosis $100 \%$ vs. T. harzianum dosis $150 \%$ \\
\hline 9 & B. subtilis dosis $100 \%$ vs. B. subtilis dosis $150 \%$ \\
\hline
\end{tabular}

* Únicos contrastes realizados en la fase de laboratorio 


\section{RESULTADOS Y DISCUSIÓN}

\section{Fase de laboratorio}

Los datos obtenidos de la evaluación diaria del crecimiento micelial de Pythium en las cajas Petri con CMA (Fig. 2) más los productos biológicos y químicos sometidos al Anova en los días 1 y 2 , con un coeficiente de variación de $5,1 \%$ y $12,0 \%$, respectivamente, mostraron diferencias significativas en el crecimiento micelial de Pythium sp. Hubo mayor crecimiento en el testigo inoculado con $36,43 \mathrm{~cm}$ en promedio. El crecimiento rápido del micelio algodonoso aéreo de Pythium sp. en CMA probablemente fue debido a la alta capacidad de desarrollo de estructuras patogénicas de este seudohongo que se han encontrado tanto en el suelo como en plantas de crisantemo (Mostowfizadeh-Ghalamfarsa y Banihashemi, 2005), esta capacidad está determinada por la disponibilidad de enzimas celulíticas y pectolíticas, las cuales se han encontrado en muchas especies (Van der Plaats-Niterink, 1981).

En el $3^{\text {er }}$ y $4^{\text {to }}$ día los análisis mostraron diferencias significativas y un coeficiente de variación de $12 \%$ y $14 \%$, respectivamente, en el crecimiento micelial de Pythium sp. en el medio de cultivo. Hubo mayor crecimiento en el tratamiento con $T$. harzianum para las dosis $150 \%$, seguido del testigo inoculado. Los tratamientos con fungicidas químicos en dosis 100\% y $150 \%$, presentaron una inhibición total del patógeno ya que no hubo crecimiento micelial de Pythium sp. en la prueba in vitro (Fig. 3). Moorman et al. (2004) mencionan que la mezcla de metalaxil y clorotalonil mostró un excelente resultado debido al buen control del fungicida de contacto (clorotalonil) y a un probable efecto sinérgico derivado de su combinación con metalaxil (sistémico), siendo uno de los mejores en el control de Oomycetes como Phytophthora infestans (Mont.) de Bary y Pythium sp., ya que posee una acción antiesporulante, inhibiendo completamente el crecimiento micelial en plantas $y$, azoxystrobin es una estrobilurina de acción sistémica. Al respecto, Wang et al. (2016) reportaron que el efecto en medio de cultivo es debido al colapso del micelio promovido por la inhibición o interrupción de la esporulación en algunas etapas vitales del desarrollo de Pythium sp. También, se ha reportado actividad inhibidora de azoxystrobin, ya que evita la germinación de urediosporas de Puccinia hemerocallidis Thüm., e inhibe el crecimiento micelial y la esporulación de Botryosphaeria parva Pennycook \& Samuels y Phomopsis sp. (Everett et al., 2005).

Sin embargo, en el $2^{\text {do }}$ día se observó una disminución leve en el crecimiento (diámetro del micelio) en los tratamientos en donde se aplicó B. subtilis, ya que presentó una competencia directa por espacio y nutrientes (Fig. 3), la cual ha sido reportada por Ghasemi et al. (2010); mientras que, los tratamientos con aplicación de T. harzianum mostraron una disminución en la velocidad de crecimiento, probablemente debido a mecanismos de desplazamiento del antagónico hacia el fitopatógeno por antibiosis, parasitismo, competencia de espacio y nutrientes (Sid Ahmed et al., 2003).
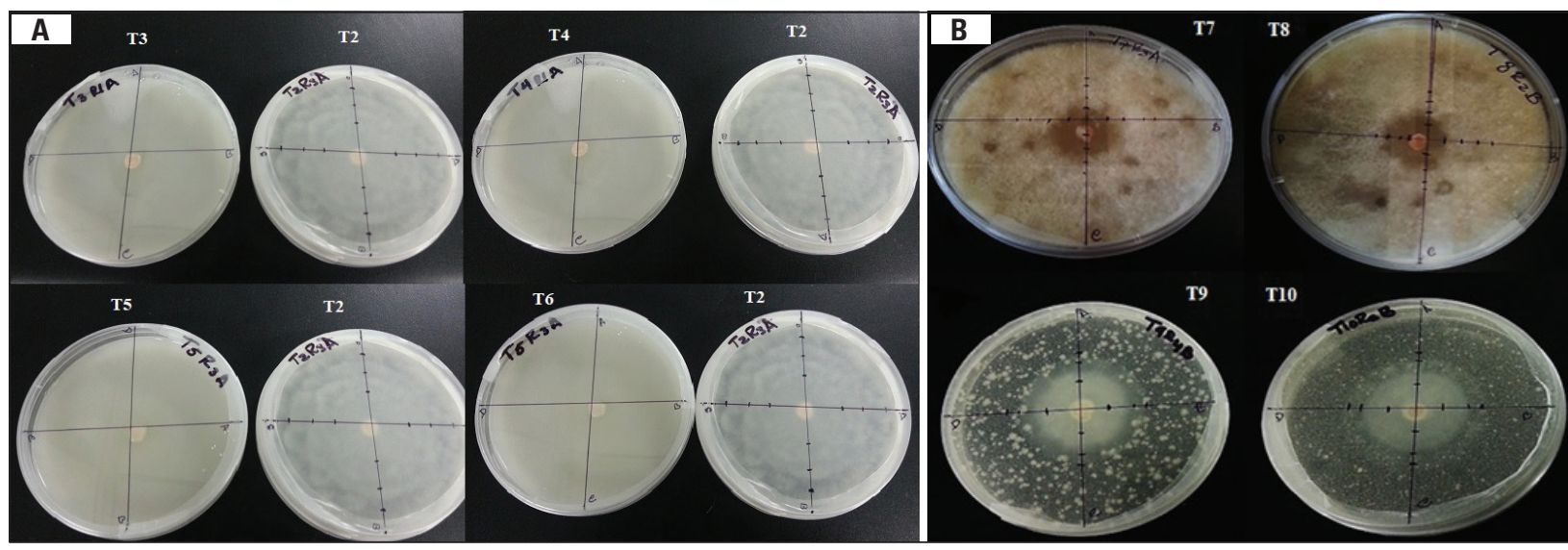

Figura 2. Inhibición del crecimiento micelial de Pythium sp. en medio CMA. A) Diferentes tratamientos químicos vs. testigo con Pythium sp. (T2): metalaxil + clorotalonil en dosis de 100\% (T3), metalaxil + clorotalonil en dosis de $150 \%$ (T4), azoxystrobin en dosis de $\mathbf{1 0 0 \%}$ (T5) y azoxystrobin en dosis de $\mathbf{1 5 0 \%}$ (T6). B) Tratamientos biológicos: $T$. harzianum en dosis de $100 \%$ (T7), T. harzianum en dosis de $150 \%$ (T8), B. subtilis en dosis de $100 \%$ (T9) y B. subtilis dosis $150 \%$ (T10). 


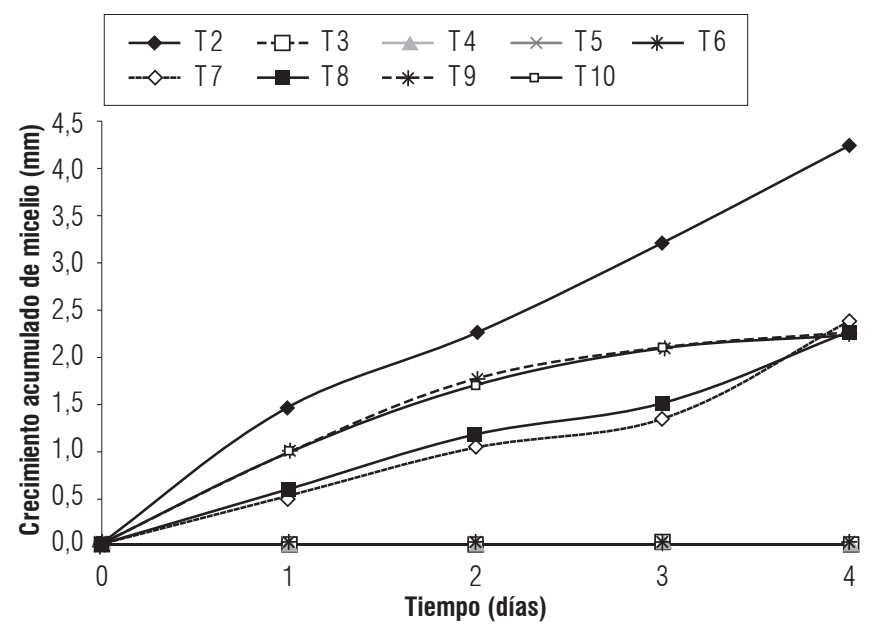

Figura 3. Crecimiento micelial de Pythium sp. en medio de CMA durante 4 días. T2: con Pythium, T3: metalaxil + clorotalonil en dosis de 100\%, T4: metalaxil + clorotalonil en dosis de 150\%, T5: azoxystrobin en dosis de 100\%, T6: azoxystrobin en dosis de 150\%, T7: T. harzianum en dosis de 100\%, T8: $T$. harzianum en dosis de 150\%, T9: B. subtilis en dosis de $100 \%$, T10: B. subtilis en dosis de $150 \%$.

Entre el día 2 y 3 hubo un aumento de crecimiento de Pythium en el testigo inoculado, mientras que disminuyó el crecimiento micelial en los tratamientos con T. harzianum y B. subtilis (Fig. 2).

El testigo inoculado con Pythium sp. mantuvo las más altas tasas de crecimiento del patógeno el día 4, mientras que los tratamientos con aplicaciones de $T$. harzianum en dosis de $100 \%$ y $150 \%$, tuvieron un crecimiento significativo (Fig. 3). Sin embargo, $2 \mathrm{~d}$ después de haber terminado la medición del crecimiento micelial del patógeno se observó un crecimiento acelerado de T. harzianum en los tratamientos donde fue aplicado, hasta observarse el cubrimiento total de la superficie del medio contenido en las cajas de Petri, atribuido a la función de antibiosis que inhibe la actividad ribosomal de los fitopatógenos disminuyendo su reproducción por medio de la micotoxina trichodermin (Moo-Koh et al., 2014).

En la evaluación de los contrastes ortogonales para los días 1,2 y 4 se observaron diferencias altamente significativas para las tres comparaciones realizadas (Tab. 3), el crecimiento micelial en los tratamientos que recibieron aplicación y los que no tuvieron aplicación mostró un promedio de 0,3234 y 1,095 cm, respectivamente, lo que significa que la aplicación de cualquier producto disminuyó el crecimiento del $P y$ thium sp.; del mismo modo, al comparar los productos de síntesis química contra los biológicos, el mejor tratamiento fue el químico, ya que inhibió totalmente el crecimiento del patógeno. Al comparar los tratamientos biológicos entre sí, el mejor efecto en el crecimiento de Pythium sp. fue con T. harzianum en dosis de $150 \%$, debido a que mostró antibiosis, parasitismo y competencia por espacio en el medio de cultivo (Sid Ahmed et al., 2003).

\section{Fase de invernadero}

Incidencia: se encontró mayor incidencia de la enfermedad en las plantas inoculadas $(66,6 \%)$, seguido del azoxystrobin en dosis de 150\% (25,3\%) mostrando un control bajo (Fig. 4); Sin embargo, la dosis de $100 \%$ tuvo un mayor control de los síntomas de la enfermedad (17,3\%), lo anterior puede ocurrir debido a que las estrobilurinas tienden a tener un sitio de acción más específico y por lo tanto es más fácil que el patógeno se haga resistente en dosis muy altas, aun así Rebollar-Alviter et al. (2005) mencionan que los fungicidas a base de estrobilurinas están registrados para el control de varias enfermedades entre ellas las causadas por oomycetes en diferentes cultivos, sin embargo, Pythium sp. no está reportado en crisantemo.

Las dosis de T. harzianum presentaron efectividad alta con solo $9,33 \%$ de incidencia de la enfermedad (Fig. 4), evidenciando antagonismo a Pythium sp. en la raíz, lo que genera inducción de resistencia sistémica a la planta, mayor absorción y solubilidad 


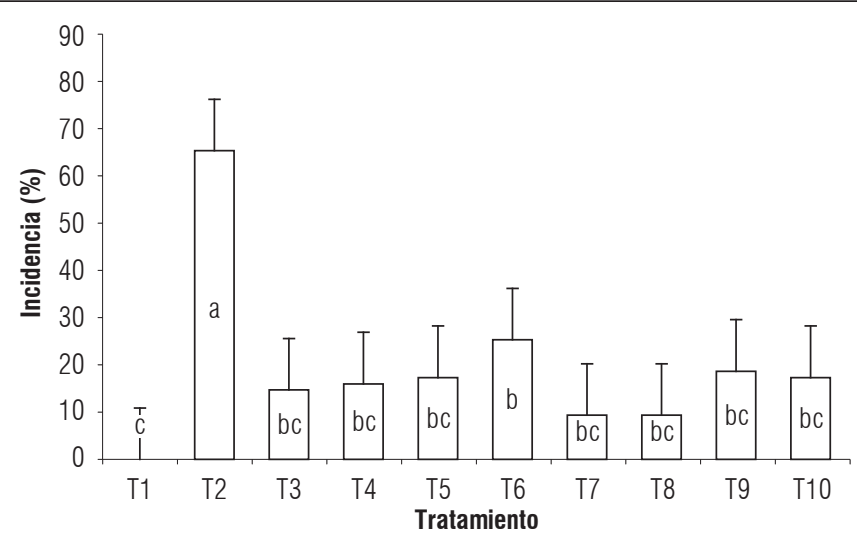

Figura 4. Incidencia del ahogamiento de plántulas de crisantemo causado por Pythium sp. T1: sin Pythium, T2: con Pythium, T3: metalaxil + clorotalonil en dosis de $100 \%$, T4: metalaxil + clorotalonil en dosis de $150 \%$, T5: azoxystrobin en dosis de 100\%, T6: azoxystrobin en dosis de 150\%, T7: T. harzianum en dosis de 100\%, T8: T. harzianum en dosis de $150 \%$, T9: $B$. subtilis en dosis de $100 \%$, T10: $B$. subtilis en dosis de $150 \%$. Letras distintas indican diferencias significativas entre tratamientos según la prueba de Tukey $(P \leq 0,05)$. Las barras sobre las columnas indican el error estándar $(n=5)$.

de nutrientes, desarrollo radicular y enraizamiento (Harman, 2006), lo que permitió obtener flores de corte de buena calidad, en cuanto al diámetro de flor abierta con un promedio de $82,6 \mathrm{~mm}$ y con una masa floral de 3,5 g. La mezcla metalaxil + clorotalonil presentó menor eficacia probablemente a la alta solubilidad y movilidad del producto en el suelo, lo cual favorece la lixiviación rápida del ingrediente $y$ por ende presente bajas concentraciones durante el período crítico de ocurrencia de la enfermedad, lo que limita la protección requerida (50 d después de la inoculación del fitopatógeno) (Rebollar-Alviter et al., 2005).

El Anova mostró diferencias significativas para la incidencia, lo que indica que los tratamientos usados controlaron en diferente grado la enfermedad. Sin embargo, los tratamientos con T. harzianum tuvieron mejor respuesta en comparación con azoxystrobin, lo cual se atribuye a la habilidad que tiene T. harzianum para colonizar las raíces y crecer con mayor facilidad en el suelo, mientras que el fungicida se lixivia con el riego (Durman et al., 2003). Además, se ha reportado que T. harzianum es capaz de producir sideróforos que intervienen en el proceso de oxidación, solubilización, quelatación y reducción de $\mathrm{Fe}, \mathrm{Fe}^{3+}$ y $\mathrm{Cu}^{2+}$, elementos importantes en el desarrollo del Pythium sp. (Cano, 2011). Se observaron diferencias estadísticas altamente significativas entre los tratamientos con la evaluación de contrastes ortogonales, mostrando, como era de esperarse, menor incidencia de la enfermedad en los tratamientos con aplicación; esto indica que es necesario el uso de algún tipo de control, ya que Pythium sp. coloniza las raíces de las plantas causando la disminución de la calidad en los tallos y flores, y finalmente pérdida total de las plantas de crisantemo (Xue et al., 2015).

Severidad: Se observó que las dosis de 100\% y 150\% de T. harzianum tuvieron un control estadísticamente significativo en la reducción de la severidad de la enfermedad, expresado en menor número de plantas enfermas (Fig. 5). Las plantas del testigo inoculadas con Pythium sp. tuvieron una severidad del 100\% (Fig. 5), lo cual causó la muerte de todas las plantas. No obstante, el desarrollo de las distintas especies de Pythium sp. puede variar de unas zonas a otras, debido a la capacidad de adaptación del microorganismo a diversas condiciones climáticas (Pérez et al., 2011). A diferencia de T. harzianum, azoxystrobin en dosis de $150 \%$ obtuvo un control reducido de la enfermedad, con una severidad final $84,8 \%$.

El Anova de la severidad presentó diferencias significativas, esto indica que los tratamientos aplicados tuvieron efecto en el control de la enfermedad. Al observar las raíces de las plantas con el inóculo de $P$. aphanidermatum, éstas mostraron una coloración café oscura a negro, con lesiones de pudrición húmeda y un área radicular reducida, mientras que las raíces inoculadas con T. harzianum en dosis 150\% mostraron mayor desarrollo radicular con coloración clara y sin pudrición, atribuida a la estimulación que produce Trichoderma en la aceleración del desarrollo 


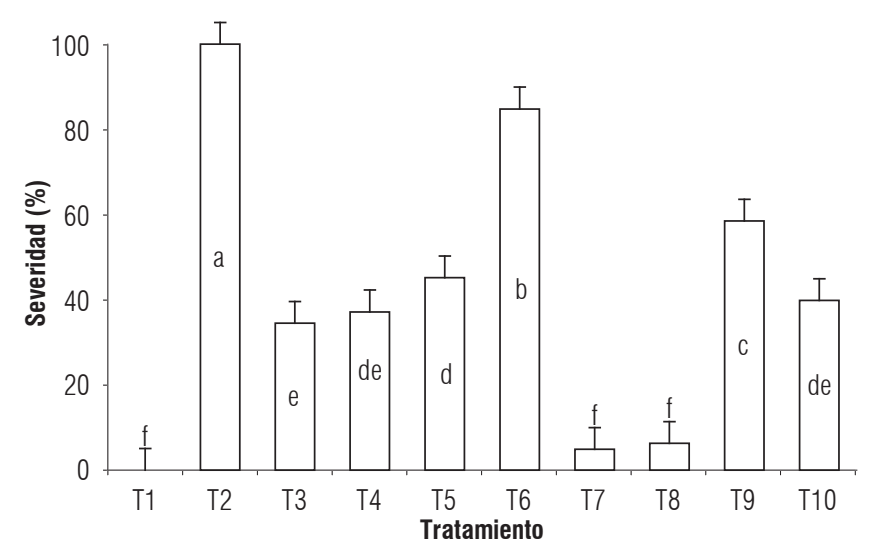

Figura 5. Severidad del ahogamiento de plántulas de crisantemo causado por Pythium sp. T1: sin Pythium, T2: con Pythium, T3: metalaxil + clorotalonil en dosis de $100 \%$, T4: metalaxil + clorotalonil en dosis de $150 \%$, T5: azoxystrobin en dosis de $100 \%$, T6: azoxystrobin en dosis de $150 \%$, T7: T. harzianum en dosis de 100\%, T8: $T$. harzianum en dosis de $150 \%$, T9: $B$. subtilis en dosis de $100 \%$, T10: $B$. subtilis en dosis de $150 \%$. Letras distintas indican diferencias significativas entre tratamientos según la prueba de Tukey $(P \leq 0,05)$. Las barras sobre las columnas indican el error estándar $(n=5)$.

del sistema radicular que posibilita la tolerancia de la planta a diferentes fitopatógenos (Harman, 2006). La evaluación de los contrastes mostró diferencias estadísticas altamente significativas para la severidad de la enfermedad en la mayoría de los tratamientos.

Características agronómicas: respecto a la altura de las plantas, el Anova mostró diferencias significativas entre tratamientos, donde T. harzianum en dosis de 150\% mostró el mejor desarrollo con $69 \mathrm{~cm}$ de longitud de vara promedio (Fig. 6A), probablemente debido a la capacidad de Trichoderma de producir sustancias estimuladoras al crecimiento vegetal (Harman, 2006).

La masa fresca de raíces presentó diferencias significativas entre tratamientos, obteniendo $9,39 \mathrm{~g}$ para $T$. harzianum en dosis $150 \%$ (Fig. 6B). Este beneficio de Trichoderma sobre la masa radicular se debe a su desarrollo en modo de cobertura, produciendo sustancias estimuladoras del crecimiento, las cuales aceleran el crecimiento de las partes jóvenes de las raíces y activan la división celular (Bae y Knudsen, 2005).

El Anova para la masa seca de raíces mostró diferencias significativas. El tratamiento T. harzianum dosis $150 \%$ produjo la mayor masa (2,54 g, Fig. 6C), lo que resalta el efecto de este biocontrolador sobre el desarrollo de las raíces, al propiciar mayor acumulación de biomasa seca como lo reporta Ghorbanpoura et al. (2018).
En cuanto a la masa fresca aérea no se obtuvieron diferencias estadísticas significativas. El tratamiento $T$. harzianum dosis $150 \%$ presentó los mayores valores (63,3 g, Fig. 6D), lo cual concuerda con los resultados obtenidos en masa radicular fresca y seca, que permite inferir un mayor desarrollo de raíces se obtendrá mejor crecimiento de la parte aérea de las plantas, principalmente en el transporte de nutrientes, además, T. harzianum produce niveles endógenos de giberelinas y citoquininas, las cuales promueven el crecimiento longitudinal de los tallos y modifican el crecimiento de las plantas (Harman, 2006).

Sobre la masa seca aérea, el tratamiento T. harzianum dosis $150 \%$ mostró diferencias significativas respecto a los demás tratamientos (28,8 g, Fig. 7A), lo cual demuestra la importancia de Trichoderma como biocontrolador de fitopatógenos y promotor de crecimiento al producir fitohormonas que inducen la producción de brotes en la parte aérea de las plantas de crisantemo (Cubillos-Hinojosa et al., 2009), y por consiguiente, mayor masa fresca aérea.

Los contrastes ortogonales revelaron diferencias altamente significativas entre las comparaciones para las variables altura de las plantas, masa fresca y seca de raíces y parte aérea, lo que permite apreciar la importancia de la aplicación de algún tratamiento para controlar la enfermedad (Tab. 4). 


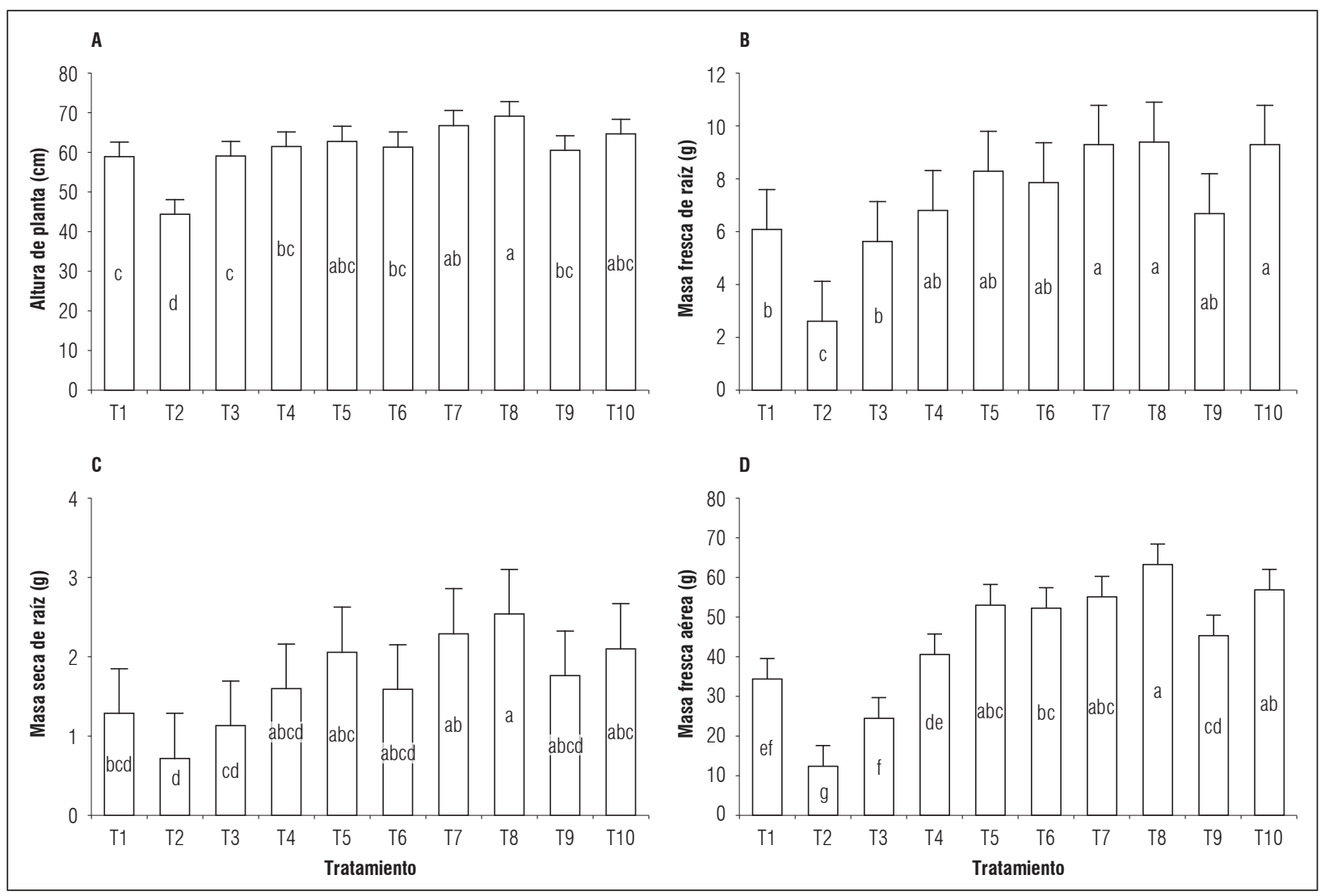

Figura 6. A) altura, B) masa fresca de raíz, C) masa seca de raíz, y D) masa fresca aérea de crisantemo con tratamientos a Pythium sp. T1: sin Pythium, T2: con Pythium, T3: metalaxil + clorotalonil dosis $100 \%$, T4: metalaxil + clorotalonil dosis 150\%, T5: azoxystrobin dosis 100\%, T6: azoxystrobin dosis $150 \%$, T7: T. harzianum dosis $100 \%$, T8: T. harzianum dosis $150 \%$, T9: $B$. subtilis dosis $100 \%$, T10: $B$. subtilis dosis $150 \%$. Letras distintas indican diferencias significativas entre tratamientos según la prueba de Tukey $(P \leq 0,05)$. Las barras sobre las columnas indican error estándar $(n=5)$.

Tabla 4. Valores de probabilidad del Anova para los contrastes ortogonales en tratamientos a Pythium sp. en crisantemo.

\begin{tabular}{|c|c|c|c|c|c|}
\hline Comparaciones & AP & MFR & MFS & MFA & MSA \\
\hline Con aplicación de control vs. Sin aplicación & $<0,0001$ & $<0,0001$ & $<0,0001$ & $<0,0001$ & $<0,0001$ \\
\hline Testigo sin Pythium sp. vs. Testigo con Pythium sp. & $<0,0001$ & 0,0004 & 0,1010 & $<0,0001$ & 0,0777 \\
\hline Tratamientos químicos vs. Tratamientos biológicos & 0,0007 & 0,0016 & 0,0014 & $<0,0001$ & $<0,0001$ \\
\hline Metalaxil + clorotalonil vs. Azoxystrobin & 0,2454 & 0,0055 & 0,0607 & $<0,0001$ & 0,3900 \\
\hline Azoxystrobin dosis $100 \%$ vs. Azoxystrobin dosis $150 \%$ & 0,5147 & 0,6216 & 0,1703 & 0,7969 & 0,4370 \\
\hline $\begin{array}{l}\text { Metalaxil + clorotalonil dosis } 100 \% \text { vs. Metalaxil + clorotalonil } \\
\text { dosis } 150 \%\end{array}$ & 0,3019 & 0,1952 & 0,1702 & $<0,0001$ & 0,0318 \\
\hline Trichoderma harzianum vs. Bacillus subtilis & 0,0015 & 0,0393 & 0,0502 & 0,0007 & $<0,0001$ \\
\hline T. harzianum dosis $100 \%$ vs. T. harzianum dosis $150 \%$ & 0,3047 & 0,9024 & 0,4696 & 0,0124 & 0,0027 \\
\hline B. subtilis dosis $100 \%$ vs. B. subtilis dosis $150 \%$ & 0,0722 & 0,0062 & 0,3207 & 0,0006 & 0,0033 \\
\hline
\end{tabular}

AP: altura de la planta; MFR: masa fresca de raíces; MFS: masa seca de raíces; MFA: masa fresca aérea; y MSA: masa seca aérea. Valores de probabilidad menores a 0,05 indican diferencias significativas, valores de probabilidad menores 0,01 indican diferencias altamente significativas. 
El tratamiento T. harzianum dosis $150 \%$ presentó el mayor rendimiento para el número de botones florales, diámetro y masa floral con promedio de 14,4 botones florales, $82,6 \mathrm{~mm}$ de diámetro y $3,5 \mathrm{~g}$ de masa floral (Fig. 7B, 7C y 7D). La aplicación de T. harzianum mostró los mejores resultados sobre dichas variables debido a que este hongo, estimula la producción de citoquininas, las cuales promueven la división y diferenciación celular, mientras que las giberelinas promueven el crecimiento y desarrollo floral (Cubillos-Hinojosa et al., 2009). La evaluación estadística de contrastes ortogonales reveló diferencias estadísticas altamente significativas para las variables número de botones florales, diámetro y masa floral para las combinaciones: con aplicación y sin aplicación de algún control, testigo sin y con Pythium sp., control químico y biológico y para los tratamientos T. harzianum y B. subtilis. Se destacan los resultados con T. harzianum en la promoción del crecimiento y desarrollo del cultivo de crisantemo por la producción de metabolitos que estimulan los procesos de desarrollo vegetal (Harman, 2006), entre ellos, la formación del botón floral y la apertura del mismo, razón por la cual se encontraron tallos con mayor diámetro y masa de flor.

La correlación de variables mostró proporcionalidad directa entre incidencia y severidad indicando que a mayor incidencia más severidad de la enfermedad (Fig. 8). Además, una relación inversa entre incidencia y severidad en comparación con las variables agronómicas: altura de planta (longitud de tallo), masa fresca y seca de raíz y parte aérea, número de botones florales, diámetro y masa de flor, lo cual permite inferir que la mayor presencia de la enfermedad afecta directamente la calidad de la flor.

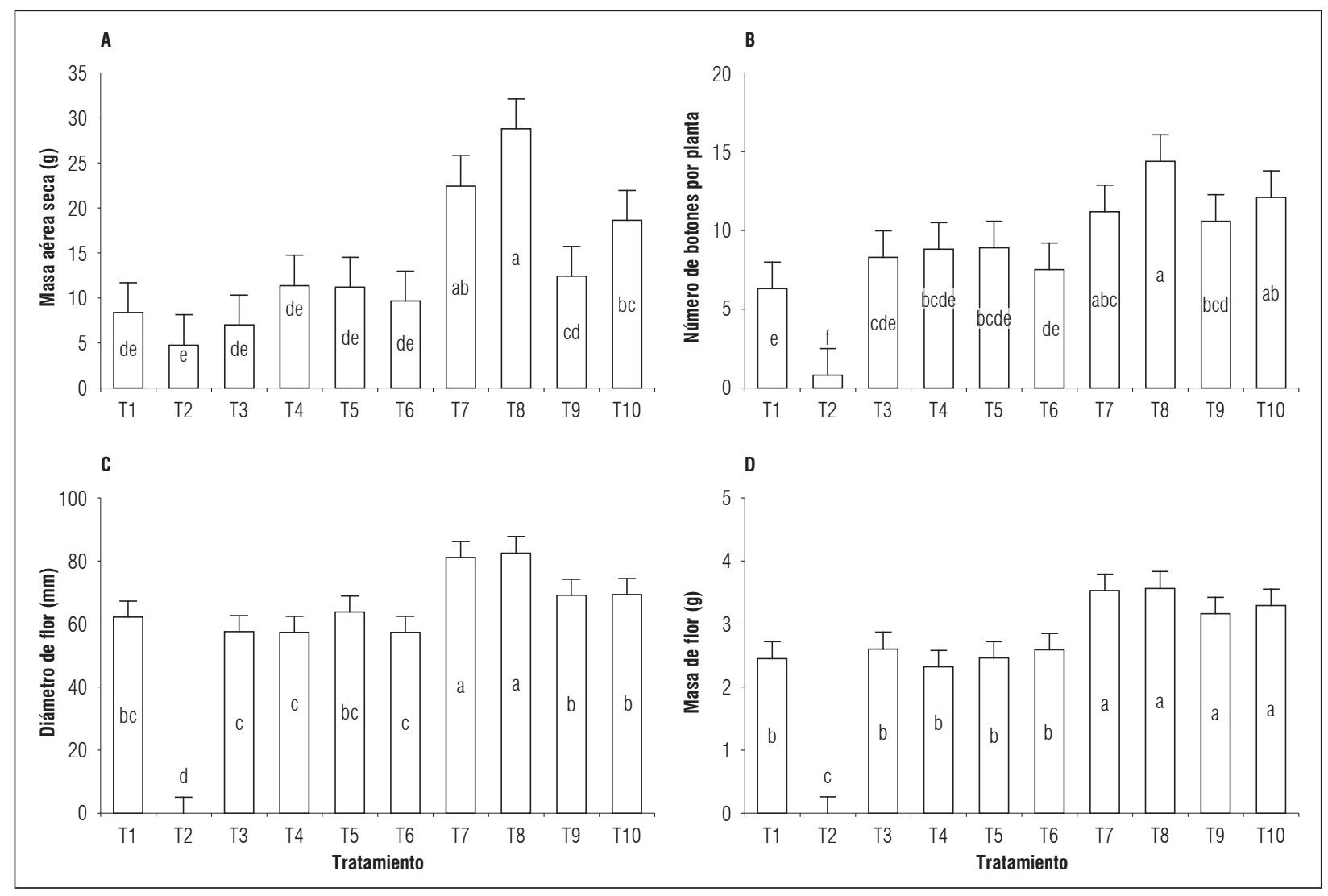

Figura 7. Evaluación de A. masa seca aérea; B. número de botones; C. diámetro de flor, masa de flor en plantas de crisantemo. T1: Sin Pythium, T2: con Pythium, T3: Metalaxil + Clorotalonil en dosis de 100\%, T4: Metalaxil + Clorotalonil en dosis de 150\%, T5: Azoxystrobin en dosis de 100\%, T6: Azoxystrobin en dosis de 150\%, T7: T. harzianum en dosis de 100\%, T8: T. harzianum en dosis de 150\%, T9: B. subtilis en dosis de $100 \%$, T10: $B$. subtilis en dosis de $150 \%$. Letras distintas indican diferencias significativas entre tratamientos según la prueba de Tukey $(P \leq 0,05)$. Las barras verticales indican el error estándar $(n=5)$. 


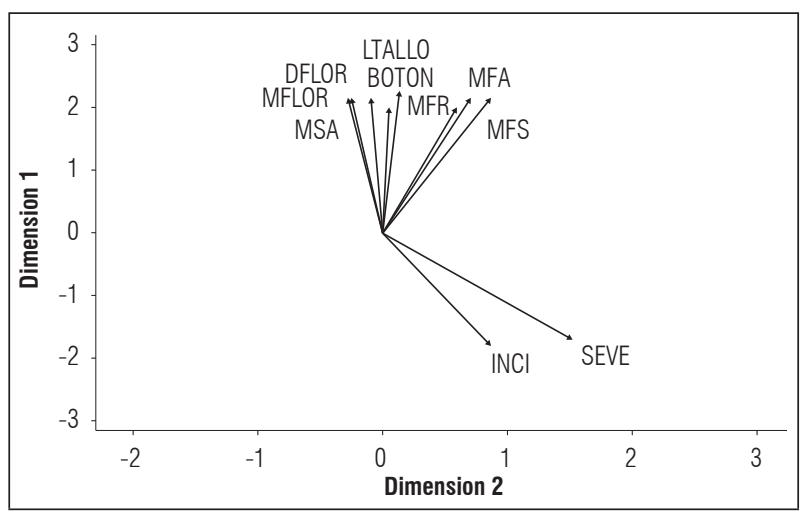

Figura 8. Correlación de variables en plantas de crisantemo con tratamientos a Pythium sp. MFR: masa fresca de raíces; MFS: masa seca de raíces; MFA: masa fresca aérea; y MSA: masa seca aérea; INCI: incidencia; SEVE: severidad. LTALLO: longitud del tallo floral; BOTON: número de botones florales; DFLOR: diámetro de flor; MFLOR: masa de flores.

\section{CONCLUSIONES}

La dosis $0,01 \mathrm{~g} / 201 \mathrm{~mL}$ de $T$. harzianum fue la más efectiva en evaluaciones de laboratorio en comparación con B. subtilis. La dosis T. harzianum 150\% tuvo el mejor control sobre Pythium sp. en plantas de crisantemo bajo condiciones de invernadero. La dosis de $0,015 \mathrm{~mL} / 0,33 \mathrm{~mL}$ de metalaxil + clorotalonil fue más efectiva en comparación con las dosis de 0,023 $\mathrm{g} / 59 \mathrm{~mL}$ y $0,0352 \mathrm{~g} / 117 \mathrm{~mL}$ de azoxystrobin. El mayor control de Pythium sp. se obtuvo con T. harzianum en dosis de $150 \%$ y mostró los mejores resultados entre tratamientos y variables evaluados.

Conflicto de intereses: el manuscrito fue preparado y revisado con la participación de los autores, quienes declaran no tener algún conflicto de interés que coloquen en riesgo la validez de los resultados aquí presentados.

\section{REFERENCIAS BIBLIOGRÁFICAS}

Bae, Y.S. y G. Knudsen. 2005. Soil microbial biomass influence on growth and biocontrol efficacy of Trichoderma harzianum. Biol. Control 32, 236-242. Doi: 10.1016/j.biocontrol.2004.10.001

Cano, M.A. 2011. Interacción de microorganismos benéficos en plantas: micorrizas, Trichoderma spp. y Pseudomonas spp. Una revisión. Rev. U.D.C.A Act. \& Div. Cient. 14(2), 15-31.
Cubillos-Hinojosa, J., N. Valero y L. Mejía. 2009. Trichoderma harzianum como promotor del crecimiento vegetal del maracuyá (Passiflora edulis var. flavicarpa Degener). Agron. Colomb. 27(1), 81-86.

Dhingra, O.D. y B. Sinclair. 1995. Basic plant pathology methods. CRC Press, Cleveland, OH. pp. 31-380.

Durman, S., A. Menéndez y A. Godeas. 2003. Evaluación de Trichoderma spp. como antagonista de Rhizoctonia solani "in vitro" y como biocontrolador del damping off de plantas de tomate en invernadero. Rev. Argent. Microb. 31(1), 13-18.

Everett, K.R., S.G. Owen y J.G. Cutting. 2005. Testing efficacy of fungicides against post-harvest pathogens of avocado (Persea americana cv. Hass). New Z. J. Plant Prot. 58, 89-95.

Ghasemi, S., G. Ahmadian, N. Jelodar, H. Rahimian, S. Ghandili, A. Dehestani y P. Shariati. 2010. Antifungal chitinases from Bacillus pumilus SG2: preliminary report. World J. Microbiol. Biotechnol. 26, 1437-1443. Doi: 10.1515/biolog-2015-0112

Ghorbanpoura, M., M. Omidvarib, P. Abbaszadeh-Dahajic, R. Omidvard y K. Karimane. 2018. Mechanisms underlying the protective effects of beneficial fungi against plant diseases. Biol. Control 117, 147-157. Doi: 10.1016/j.biocontrol.2017.11.006

Harman, G. 2006. Overview of mechanisms and uses of Trichoderma spp. Phytopathology 96(2), 190-194. Doi: 10.1094/PHYTO-96-0190

Henfling, J.W. 1982. Field screening procedures to evaluate resistance to late blight. International Potato Center (CIP), Lima.

León, J. 2017. Minagri busca promover producción y comercialización de flores. En: Agraria.pe, http://www. agraria.pe/noticias/minagri-busca-promover-produccion-y-comercializacion-13923; consultado: noviembre de 2017.

Moo-Koh, F., C. Alejo, A. Reyes-Ramírez, M. Tun-Suarez, R. Sandoval-Luna y A. Ramirez-Pool. 2014. Actividad in vitro del extracto acuoso Bonella flammea contra hongos fitopatógenos. Agrociencia 48, 833-845.

Moorman, G., S. Kang, D. Geiser y S. Kim. 2004. Identification and characterization of Pythium species associated with greenhouse floral crops in Pennsylvania. Plant Dis. 86, 1227-1231. Doi: 10.1094/PDIS.2002.86.11.1227

Mostowfizadeh-Ghalamfarsa, R. y Z. Banihashemi. 2005. Identification of soil Pythium species in farms province of Iran. Iranian J. Sci. Technol. Trans. A 29(A1), 79-87.

Pérez, A., J. Rojas y D. Montes. 2011. Hongos formadores de micorrizas arbusculares, una alternativa biológica para la sostenibilidad de los agroecosistemas de praderas en El Caribe colombiano. Rev. Colomb. Cienc. Animal 3(2), 366-385.

Rebollar-Alviter, A., L.V. Madden y M.A. Ellis. 2005. Efficacy of azoxystrobin, pyraclostrobin, potassium phosphite 
and mefenoxam for control of strawberry leather rot caused by Phytophthora cactorum. Plant Health Prog. 12, 67-68. Doi: 10.1094/PHP-2005-0107-01-RS

Rinaudo, V., P. Barberi, M. Giovannetti y M. Van Der Heijden. 2010. Mycorrhizal fungi suppress aggressive agricultural. Weeds 333, 7-20. Doi: 10.1007/ s11104-009-0202-z

Sid Ahmed, A., M. Ezziyyani, C. Pérez-Sánchez y M.E Candela. 2003. Effect of chitin on biological control activity of Bacillus spp. and Trichoderma harzianum against root rot disease in pepper (Capsicum annuum) plants. Eur. J. Plant Pathol. 109, 418-426.
Van der Plaats-Niterink, A. 1981. Monograph of the genus Pythium. Stud. Mycol. 21, 7-198.

Wang, H., Y. Huang, J. Wang, X. Chen, K. Wei, M. Wang y S. Shang. 2016. Activities of azoxystrobin and difenoconazole against Alternaria Alternata and their control efficacy. Crop Prot. 90, 54-58. Doi: 10.1016/j. cropro.2016.08.022

Xue, J., X. Chen, W. Jiang, F. Liu y H. Li. 2016. Rapid and sensitive analysis of nine fungicide residues in chrysanthemum by matrix extraction-vortex-assisted dispersive liquid-liquid microextraction. J. Chromatogr. B. Analyt Technol. Biomed. Life Sci. 975, 9-17. Doi: 10.1016/j.jchromb.2014.10.029 